\title{
Cinética de la Biodegradación de un Efluente Lácteo. Aplicación de Técnicas de Análisis de Datos para la Comparación de Modelos
}

\author{
Miguel A. Rosa, José M. Peralta, Daniel A. Gilli y Diego M. Bosco \\ Universidad Tecnológica Nacional, Facultad Regional Villa María, Grupo de Investigación \\ en Simulación para Ingeniería Química (GISIQ), Avda. Universidad 450, (X5900HLR) Villa María, \\ Córdoba-Argentina (e-mail: rosa_m@frvm.utn.edu.ar)
}

\begin{abstract}
Resumen
Se realizó un análisis comparativo de tres modelos cinéticos (Monod, exponencial y cinética de orden- $n$ ) representativos del consumo de substrato de los efluentes de una empresa láctea. Se empleó técnicas de simulación y de análisis de datos disponibles en el programa Aquasim v. 2.1.b. Para ello se correlacionaron datos experimentales obtenidos a nivel piloto, efectuándose en cada caso la estimación de los parámetros cinéticos y el análisis de sensibilidad. Se determinó que el modelo cinético de orden- $n\left(\mu=k S^{n}\right)$, resulta la opción más adecuada para representar la degradación del substrato de los efluentes. Con este modelo, además de lograr un ajuste aceptable de los datos experimentales sus parámetros $k$ y $n$ son claramente identificables. El modelo de Monod, sin embargo, aún cuando genera un menor error, contiene parámetros no identificables.
\end{abstract}

\section{Kinetic of Dairy Wastewater Biodegradation. Applications of Data Analysis Techniques for Model Comparison}

\begin{abstract}
A comparative analysis of three kinetic models (Monod, exponential and n-order kinetic) representative of substrate degradation of effluents from a dairy factory is presented. Estimation of model parameters and sensitivity analysis were done through data analysis and computational simulation using the software Aquasim v. 2.1.b. Experimental data from a pilot plant were used, estimating the kinetic parameters and performing sensitivity analysis for each case. The n-order kinetic model $\left(\mu=k S^{n}\right)$ showed to be the best option to represent the degradation of the treated wastewater. With this model, besides achieving acceptable fitting of the experimental data, its parameters $k$ and $n$ are clearly identified. The Monod model, however, although gives lower error, contains parameters with no physical meaning.
\end{abstract}

Keywords: dairy effluents, substrate degradation, kinetic models, comparative analysis 


\section{INTRODUCCIÓN}

La gran complejidad de los sistemas naturales hace muy difícil la comprensión de los mecanismos básicos que reproducen el comportamiento de los mismos, más allá de las determinaciones experimentales. Los modelos matemáticos pueden resultar de suma utilidad para efectuar tales análisis, pues contribuyen al cálculo exacto del tiempo de evolución de un sistema con este tipo de interacciones. De esta manera, la comparación de datos medidos con aquellos calculados a partir de modelos, constituye una prueba indirecta de las hipótesis formuladas acerca de tales mecanismos (Jeppsson, 1993).

Debido a que los modelos matemáticos de sistemas naturales requieren de simplificaciones del proceso real, usualmente contienen parámetros empíricos que se determinan a partir de datos experimentales. Esta razón, asociada con el creciente desarrollo de la simulación de procesos, indujo al diseño de programas de computación capaces de efectuar la simulación propiamente dicha y de juzgar la identificabilidad de los parámetros del modelo para estimar sus valores, a partir de los datos medidos y la incertidumbre de los resultados calculados (Reichert, 1998).

En el caso particular de aguas residuales de industrias lácteas se reportan importantes trabajos que consideran distintas alternativas de tratamiento en función de las características específicas del efluente. Entre los más destacados, se presenta una revisión de los avances recientes y casos de estudio para procesos anaerobios, discutiendo además métodos de tratamiento combinados anaerobios - aerobios (Demirel et al., 2005); otros autores (Vidal et al., 2000), analizan la influencia del contenido de grasas y proteínas en la biodegradación anaeróbica. Para los procesos aerobios existen también importantes trabajos de investigación referidos a: purificación aeróbica en régimen continuo (Carta-Escobar et al., 2004, 2005); sistema reactor batch secuencial - membrana (Bae et al., 2003); bases experimentales del tratamiento de barros activados para residuos industriales, estado del arte (Orhon et al., 1999), entre otros.

Cualquiera sea el caso, para llevar a cabo el diseño de los reactores biológicos resulta imprescindible conocer la cinética de las reacciones que ocurren en estos procesos con la finalidad de determinar la velocidad a la cual los microorganismos degradan un residuo específico.

En este trabajo se utilizaron las técnicas de estimación de parámetros y análisis de sensibilidad disponibles en un software específico de simulación e identificación de sistemas acuáticos (Aquasim v. 2.1.b). En este programa, las ecuaciones algebraicas discretizadas espacialmente, junto con las ecuaciones diferenciales ordinarias son integradas numéricamente en el tiempo con el algoritmo llamado Differential Algebraic System Solver (DASSL) basado en la técnica de integración de Gear. El análisis de sensibilidad combina el análisis de identificabilidad y el análisis de incertidumbre, que permite evaluar en primer término si los parámetros del modelo pueden ser determinados inequívocamente a partir de los datos disponibles y luego, calcular la incertidumbre de los parámetros durante el procedimiento de estimación (Reichert, 1998).

Sobre esta base, en el presente trabajo se realiza el estudio comparativo de tres modelos cinéticos representativos de la biodegradación aeróbica de los efluentes de una importante industria láctea de la región. Los modelos propuestos fueron: el clásico de Monod, un modelo matemático de tipo exponencial (Colazo et al., 2006) y un tercero correspondiente a una cinética de orden "n".

\section{DESARROLLO METODOLÓGICO}

Se trabajó con efluentes de una importante industria láctea regional cuya procedencia es básicamente de suero de leche, finos de queso y residuos de limpieza de la planta. El suero utilizado fue caracterizado previamente, obteniéndose resultados promedios de la DQO (demanda química de oxígeno en $\mathrm{mg}$ de $\mathrm{O}_{2} / \mathrm{L}$ ) en el orden de los $5000 \mathrm{mg}$ de $\mathrm{O}_{2} / \mathrm{L} \mathrm{y} \mathrm{pH}=7$, con valores extremos de 3 y 11 cuando es efectuada la limpieza de las instalaciones. El tratamiento se realizó en un reactor de 4 litros de capacidad, con agitación e inyección de aire permanente para mantener la condición de mezcla completa y condiciones aeróbicas. Se trabajó a temperatura ambiente, la cual osciló entre 15 ${ }^{\circ} \mathrm{C}$ y $20^{\circ} \mathrm{C}$ durante toda la experiencia. Se empleó un inóculo procedente de lodos de una laguna 
facultativa de tratamiento de efluentes urbano-domiciliarios, el cual fue aclimatado previamente durante 7 días, utilizando como sustrato un suero proveniente de esta misma industria láctea en las mismas condiciones ambientales que en el experimento. El suero bajo tratamiento presentó una DQO inicial de $3853 \mathrm{mg}$ de $\mathrm{O}_{2} / \mathrm{L}$. Para la corrida de un solo factor y una réplica, los resultados de las determinaciones de la DQO (mg de $\left.\mathrm{O}_{2} / \mathrm{L}\right)$ fueron 3853, 2130, 1468, 884, 590, 384, 376, 366, 278, 237, 198, 188 para tiempos (en días) de 0.00, 0.50, 0.69, 0.90, 1.50, 2.47, 2.92, 3.47, 9.54, 13.52, 16.56, 20.52 respectivamente. Para la determinación de la DQO se utilizó la técnica recomendada por los Standard Methods for the.Examination of Water and Wastewater (APHA, 2005)

El tratamiento de los datos experimentales se llevó a cabo utilizando el software mencionado. El análisis de sensibilidad fue realizado estimando los errores estándares y los coeficientes de correlación de los parámetros durante el procedimiento de estimación de los mismos. Como fuente adicional de información sobre la identificabilidad de los parámetros del modelo se utilizó la función de sensibilidad de uso más común, debido a que sus unidades no dependen de las del parámetro, definida por:

$\delta_{y, p}^{a, r}=p \cdot \frac{\delta y}{\delta p}$

Esta función, donde "y" representa la variable de estado y "p" los parámetros del modelo a evaluar, mide el cambio absoluto en "y" para una variación del 100\% del parámetro. La independencia de la unidad de éste permite la comparación cuantitativa del efecto de los distintos parámetros sobre la variable. Todos estos cambios son calculados sobre una aproximación lineal que puede ser construida con la ayuda de la tangente a la función $y(p)$ en " $p$ ". Para el análisis, debe tenerse en cuenta que a mayores valores de la función de sensibilidad y a diferencias más pronunciadas en la forma de la misma (dentro del rango de datos disponibles), mayor es la exactitud en la identificabilidad de los parámetros.

La derivada parcial presente en (1) puede calcularse utilizando la siguiente expresión:

$\frac{\partial y}{\partial p} \approx \frac{y \cdot\left(p_{i}+\Delta p_{i}\right)-y\left(p_{i}\right)}{\Delta p_{i}}$,

donde $\Delta \mathrm{p}_{\mathrm{i}}$ se establece como el $1 \%$ de la desviación estándar $\sigma_{\mathrm{pi}}$ del parámetro $\mathrm{p}_{\mathrm{i}}$

Los parámetros del modelo son estimados a través del parámetro $\chi^{2}$ cuya expresión representa el error entre mediciones y resultados del modelo y determinado a través de la siguiente ecuación:

$\chi^{2}(p)=\sum_{i=1}^{n}\left(\frac{y_{\text {med,i }}-y_{i}(p)}{\sigma_{\text {med }, i}}\right)^{2}$,

donde $y_{\text {med, i }}$ es la iésima medición, $\sigma_{\text {med,i }}$ es la desviación estándar, $y_{i}(p)$ es el valor calculado por el modelo para la iésima medición y evaluada para el tiempo correspondiente a esta medición, $p=\left(p_{i}\right.$, $\ldots \mathrm{p}_{\mathrm{m}}$ ) representan los parámetros del modelo y $\mathrm{n}$ es el número de determinaciones.

La minimización de la suma de la ecuación (3) puede ser realizarse a través del algoritmo simplex o el de la secante. Ambas técnicas son adecuadas para la minimización de las ecuaciones numéricamente integradas, evitando el cálculo de las derivadas parciales. 
En este trabajo, la variable $\mathrm{y}\left(\mathrm{p}_{\mathrm{i}}\right)$ corresponde a la velocidad de consumo de la DQO y su expresión depende del modelo cinético utilizado. Las siguientes son las expresiones de la velocidad de reacción correspondientes a los modelos comparados:

\section{Ecuación clásica de Monod}

$\mu=\mu_{m} \cdot \frac{S}{K_{S}+S}$,

donde $\mu$ es la velocidad de consumo específico $\left(\mathrm{mg} \cdot \mathrm{L}^{-1} \cdot \mathrm{d}^{-1}\right) ; \mu_{\mathrm{m}}$ es la velocidad máxima de consumo específica. (mg. $\left.\mathrm{L}^{-1} \cdot \mathrm{d}^{-1}\right) ; \mathrm{K}_{\mathrm{s}}$ es el coeficiente de saturación medio de substrato $(\mathrm{mg} / \mathrm{L})$ y $\mathrm{S}$ es la concentración de substrato $(\mathrm{mg} / \mathrm{L})$.

Modelo matemático de tipo exponencial decreciente

$\mu=-\frac{A_{1}}{B_{1}} \cdot e^{-\left(t-A_{0}\right) / B_{1}}-\frac{A_{2}}{B_{2}} \cdot e^{-\left(t-A_{0}\right) / B_{2}}-\frac{A_{3}}{B_{3}} \cdot e^{-\left(t-A_{0}\right) / B_{3}}$,

donde $A_{1}, A_{2}, A_{3}, B_{1}, B_{2}, B_{3}$ y $A_{0}$ son constantes empíricas cuya dependencia de variables fisicoquímicas del sistema no fue analizada. En este caso la ecuación (7) representa la derivada de la expresión matemática que correlaciona los resultados experimentales y que se obtuvo utilizando el software SPSS for Windows (Norusis, 1999; Visauta Vinacua et al., 2003).

Modelo cinético de orden " $n$ "

$\mu=k \cdot S^{n}$,

siendo $\mathrm{k}$ la constante de velocidad de reacción $\left(\mathrm{L}^{2} \cdot \mathrm{mg}^{-2} \cdot \mathrm{d}^{-1}\right)$ y $\mathrm{n}$ el orden de la reacción de biodegradación.

\section{RESULTADOS Y DISCUSIÓN}

Los resultados de la simulación y estimación de parámetros se observan en la Tabla 1 y Fig. 1. El análisis sensitivo efectuado para cada modelo arroja los resultados mostrados en las Fig. 2, 3 y 4, en las que se representa la sensibilidad absoluta-relativa, cuya expresión general viene dada por la ecuación (1) vs. tiempo. Para este caso particular, dicha función donde p representa cada uno de los parámetros del modelo, se define como:

$\delta_{y, p}^{a, r}=\frac{\partial y}{\partial p} \cdot p=\operatorname{SensAR}(D Q O, p)=\frac{\partial(D Q O)}{\partial p} \cdot p$

En la Tabla $1, \chi^{2}$ representa la suma de las desviaciones ponderadas entre valores experimentales y calculados por el modelo. 
Tabla 1: Parámetros estimados por Aquasim.

\begin{tabular}{|c|c|c|c|c|c|}
\hline Modelo, y(p) & \multicolumn{4}{|c|}{ Parámetros $(p)$} & $\chi^{2}$ \\
\hline Monod & \multicolumn{2}{|c|}{$K_{S}=53050.944$} & \multicolumn{2}{|c|}{$\mu_{\text {máx }}=57825.751$} & 2.939 \\
\hline \multirow{2}{*}{ Exponencial } & $A_{1}=1530$ & $A_{2}=35210.672$ & $A_{3}=-12509.091$ & $A_{0}=0.448$ & \multirow{2}{*}{0.069} \\
\hline & $\mathrm{B}_{1}=0.548$ & $B_{2}=-625.454$ & $B_{3}=-193.824$ & & \\
\hline Orden "n" & \multicolumn{2}{|c|}{$k=1.150 \cdot 10^{-6}$} & \multicolumn{2}{|c|}{$\mathrm{n}=2.942$} & 0.278 \\
\hline
\end{tabular}

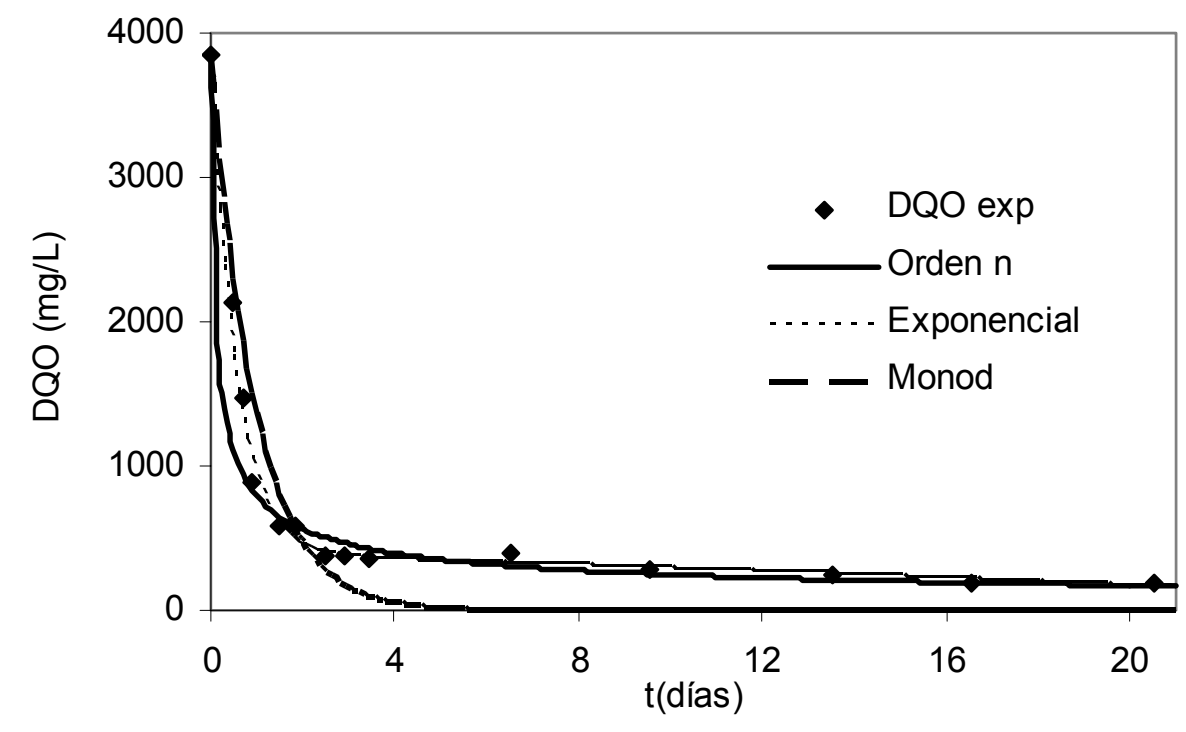

Fig. 1: Modelos vs. DQO experimental

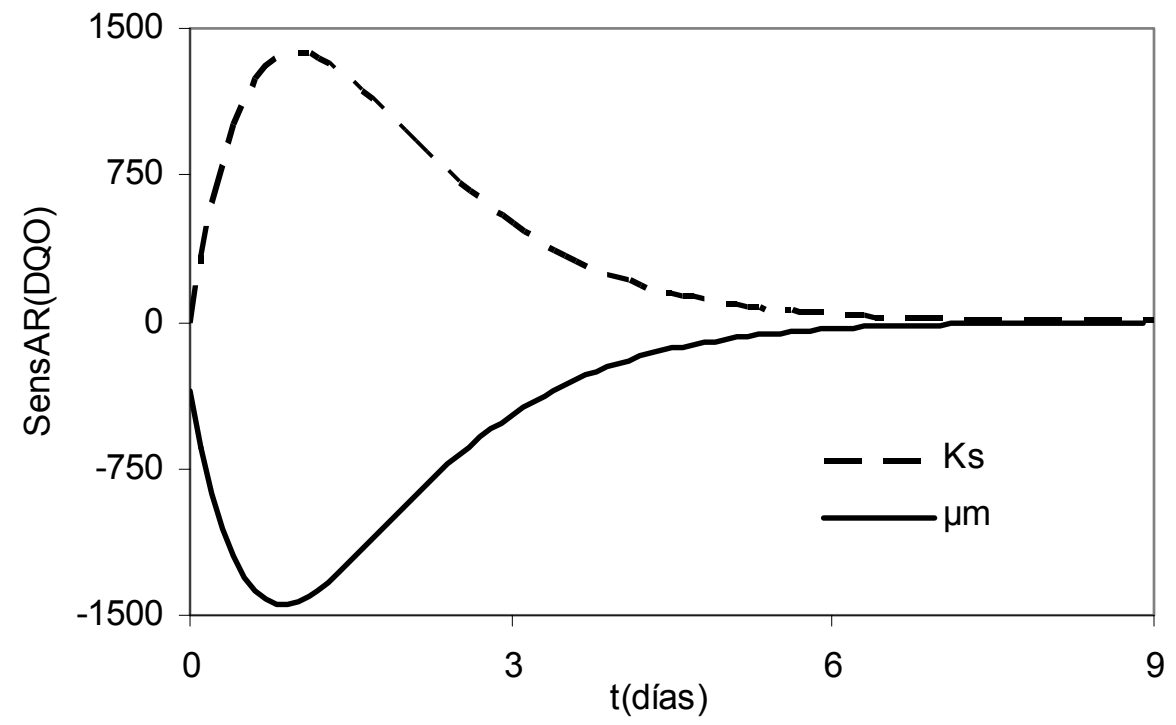

Fig. 2: Sensibilidad para el modelo Monod 


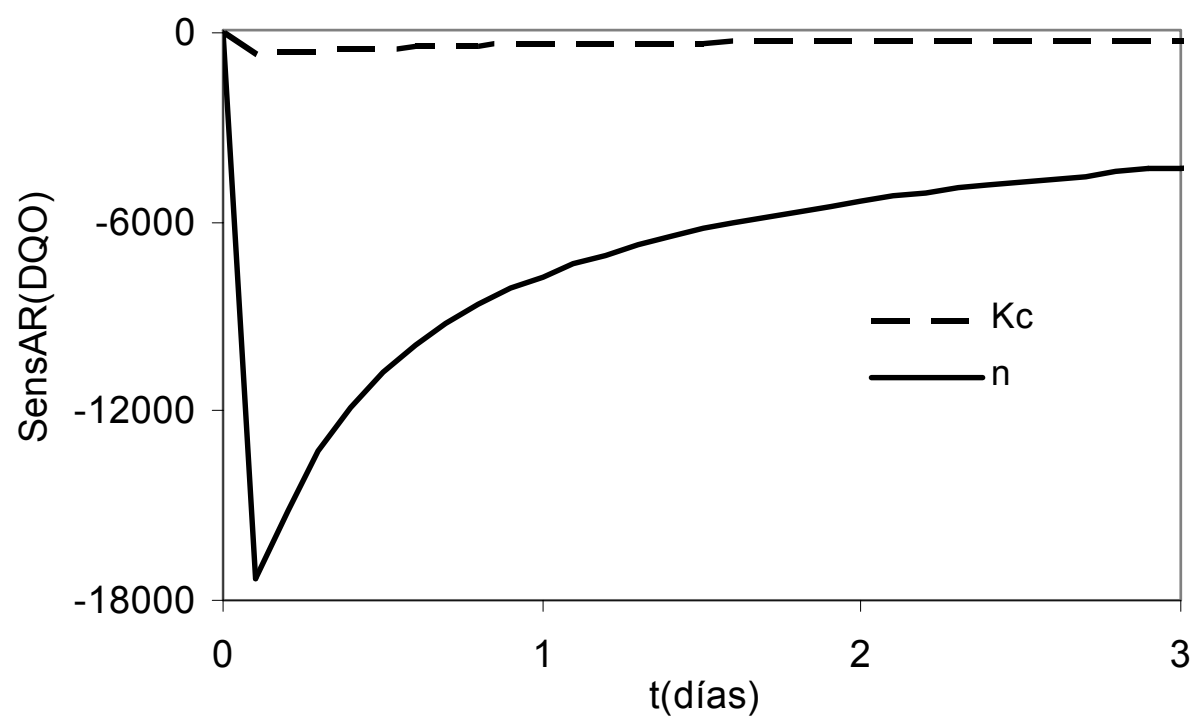

Fig. 3: Sensibilidad para el modelo de orden $\mathrm{n}$.

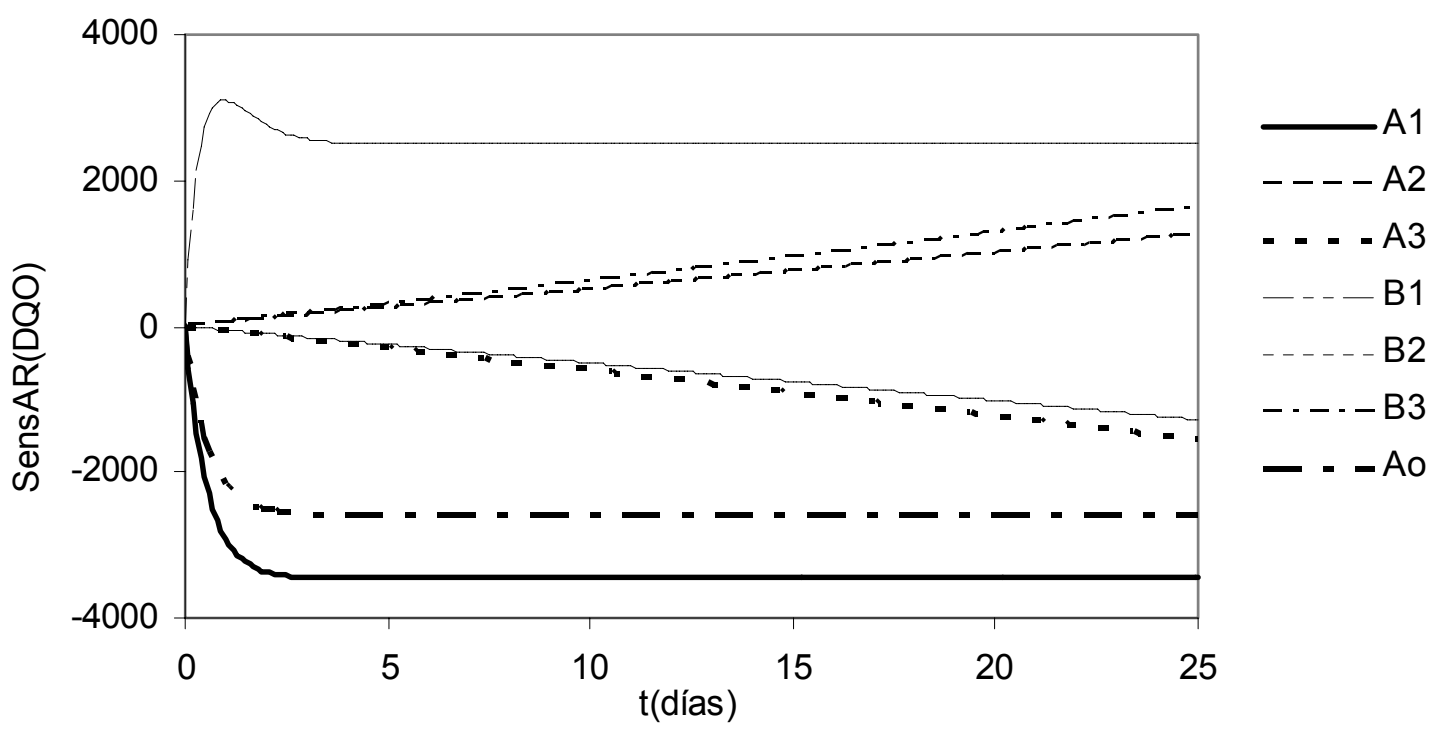

Fig. 4: Sensibilidad para el modelo exponencial.

De acuerdo con los resultados de la simulación se puede inferir que el modelo exponencial decreciente es el que mejor correlaciona los datos experimentales en base al valor final del $\chi^{2}$ obtenido, observándose además un grado de ajuste bastante aceptable en el modelo cinético de orden "n", mientras que la ecuación de Monod resulta la alternativa menos adecuada desde este punto de vista.

Para el análisis de la sensibilidad a partir de los gráficos correspondientes a los distintos modelos debe tenerse en cuenta que cuando mayores son los valores de las funciones de sensibilidad y más pronunciadas las diferencias en forma de las mismas, dentro del rango de datos procesados, la identificabilidad de los parámetros es mayor.

Del análisis de sensibilidad para cada caso, se pueden establecer las siguientes consideraciones: los parámetros del modelo de Monod resultan no identificables. Según lo observado en la Fig. 2, las funciones de sensibilidad de los parámetros $\mathrm{K}_{S}$ y $\mu_{\text {máx }}$ tienen una forma similar. Los signos indican que los valores encontrados aumentan con el incremento de valores de $\mathrm{K}$, pero disminuyen con el 
aumento de valores de $\mu_{\text {máx. }}$ Esto lleva a una correlación entre los valores estimados de estos parámetros ya que cambios en los valores calculados de DQO producen variaciones en $\mathrm{K}$ que pueden ser aproximadamente compensados por cambios apropiados de $\mu_{\text {máx. }}$. Esto permite asegurar que no existe un único par de valores $\left(\mathrm{K}_{\mathrm{S}}, \mu_{\text {máx }}\right)$ que satisfagan la expresión de Monod lo cual no garantiza la confiabilidad del modelo. Además, la similitud de las curvas de sensibilidad de ambos coeficientes, permite predecir una incertidumbre similar entre los valores estimados de ambos parámetros.

Un análisis similar, para el caso de la exponencial decreciente de la Fig. 4, permite apreciar que la concentración (DQO) es muy sensible a los parámetros $A_{0}, A_{1}$ y $B_{1}$ en prácticamente todo el intervalo de tiempo. Respecto a los restantes $\left(A_{2}, B_{2}, A_{3}\right.$ y $\left.B_{3}\right)$, la sensibilidad es muy baja para tiempos menores a 3 días, incrementándose luego de manera casi lineal. No obstante, analizando la forma de las funciones sensitivas para cada parámetro y la expresión matemática de este modelo en la cual se observa la participación en cada término de la relación $A_{i} / B_{i}$, se puede deducir, al igual que en el modelo de Monod, que variaciones de la concentración calculada pueden ser compensadas por cambios adecuados de dichos parámetros, por lo cual no son identificables y el modelo no resulta confiable.

Observando la Fig. 3, para el modelo exponencial de orden "n", se deduce que para el parámetro k la sensibilidad permanece en valores cercanos a cero durante todo el intervalo de tiempo, lo que indicaría que la incertidumbre en los valores obtenidos del mismo es importante comparada con la de los valores de $n$, para los que se aprecia una elevada sensibilidad, observándose además un comportamiento diferente de la función de sensibilidad respecto al tiempo, es decir para distintas etapas del proceso de biodegradación.

\section{CONCLUSIONES}

Se destaca la importancia de la aplicación de técnicas de análisis de datos y específicamente del análisis sensitivo para evaluar la confiabilidad de modelos cinéticos propuestos para una situación determinada.

La no identificabilidad de los parámetros correspondientes a los modelos de Monod y exponencial hacen que los mismos no resulten apropiados para representar el consumo de substrato en este caso particular, sobre todo en el modelo exponencial puesto que además los parámetros carecen de respaldo fisicoquímico.

El modelo cinético de orden "n" es considerado como la opción más adecuada entre los propuestos. El error en la estimación de los parámetros, medido a través $\chi^{2}$ es comparativamente mucho menor para este modelo cinético que para el exponencial. En el caso del modelo de Monod el error en la estimación es menor aún, pero la no identificabilidad de sus parámetros invalida su selección como modelo apropiado.

\section{REFERENCIAS}

APHA, American Public Health Association, "Standard Methods for Examination of Water and Wastewater". 21st Ed. Publication Office, American Public Health Association, New York (2005).

Bae T.H., S.S. Han y T.M. Tak; Membrane Sequencing Batch Reactor System for the Treatment of Dairy Industry Wastewater. Process Biochemistry: 39, 221-231 (2003).

Carta-Escobar F. y otro cuatro autores; Aerobic Purification of Dairy Wastewater in Continuous Regime. Part I, Biochemical Engineering. Journal: 21, 183-191 (2004).

Carta-Escobar F. y otro cuatro autores; Aerobic Purification of Dairy Wastewater in Continuous Regime. Part II, Biochemical Engineering Journal: 22, 117-124 (2005). 
Colazo C., M. Rosa, J. Peralta y G. Marín; Cinética de la Biodegradación Aeróbica de Efluentes Industriales Lácteos, Actas del XXVI Congreso Argentino de Química, San Luis, Argentina, septiembre (2006).

Demirel B., O. Yenigun y T. Okay; Anaerobic Treatment of Dairy Wastewaters: a Review, Process Biochemistry: 40, 2583-2595 (2005).

Jeppsson U.; Department of Industrial Electrical Engineering and Automation (IEA), Lund Institute of Technology, Sweden (1993).

Norusis M., SPSS Inc, Nonlinear Regression Examples, En SPSS Regression Models 10.0, 93-102, SPSS Inc., Chicago, USA (1999).

Orhon D., R. Tasli y S. Sözen; Experimental Basis of Activated Sludge Treatment for Industrial Wastewater-State of the Art, Wat. Sci. Tech.: 40(1), 23-30, (1989).

Reichert, P.; Aquasim 2.0. User Manual. Computer Program for the Identification and Simulation of Aquatic Systems. Swiss Federal Institute for Environmental Science and Technology (EAWAG), Dübendorf, Switzerland (1998).

Vidal G., A. Carvalho, R. Mendez y J.M. Lema; Influence of the Content in Fats and Proteins on the Anaerobic Biodegradability of Dairy Wastewaters, Bioresource Technol.: 74, 231-239 (2000).

Visauta Vinacua B. y I. Martori, J.C. Cañas; Modelos Avanzados de Regresión, en Análisis Estadístico con SPSS para Windows, Mc Graw Hill, Vol. II, 49-86, Madrid, España (2003). 